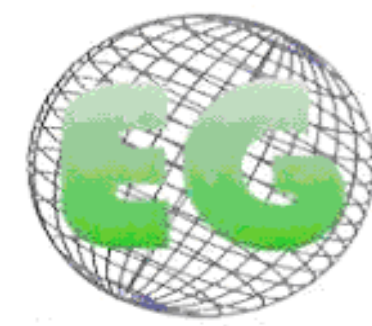

ISSN 1695-6141

$N^{\circ} 23$
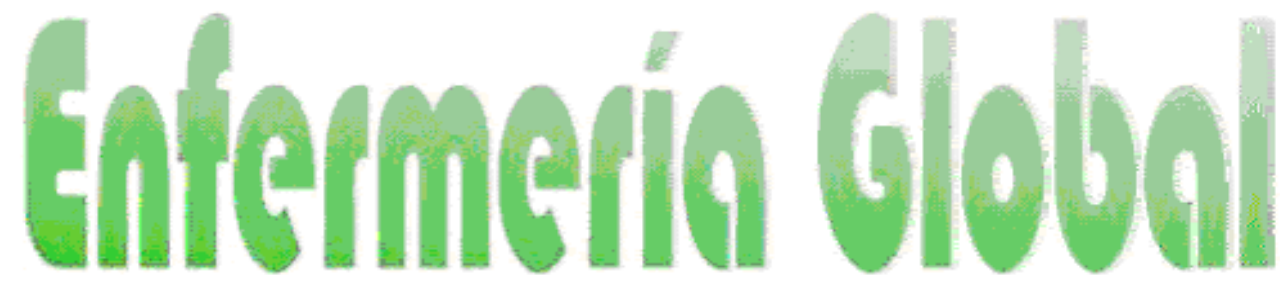

Revista electrónica trimestral de Enfermería

www.um.eslegloball

REVISIONES

\title{
Riesgos ergonómicos de lesión por esfuerzo repetitivo del personal de enfermería en el hospital
}

Riscos ergonômicos ósteo-mioesqueléticos na equipe de enfermagem em âmbito hospitalar

\author{
*De Souza, C dos S., "*Lima da Silva, JL., "**Antunes Cortez, E., "Schumacher, \\ KP., *Moreira, RCS., *De Almeida Nilson, T. \\ *Enfermeira **Professor do Departamento de Enfermagem Materno-Infantil e Psiquiátrica da Escola de \\ Enfermagem da Universidade Federal Fluminense. ${ }^{* * *}$ Doutora em Enfermagem (UFRJ). Professora do \\ Departamento de Enfermagem Materno-Infantil e Psiquiátrica da Escola de Enfermagem da Universidade \\ Federal Fluminense. Brasil
}

Palabras clave: Salud del trabajador; Riesgos laborales; Trastornos traumáticos acumulativos; Equipo de enfermería.

Palavras-chave: Saúde do trabalhador; Riscos ocupacionais; Transtornos traumáticos cumulativos; Equipe de enfermagem

Keywords: Occupational health; Occupational risks; Cumulative trauma disorders; Nursing teaming.

\section{RESUMEN}

Este estudio tuvo como objetivo identificar los riesgos ergonómicos osteo-mioesqueléticos a que se expone al personal de enfermería en los hospitales. Estudio exploratorio descriptivo llevado a cabo mediante la revisión de la literatura en los sistemas virtuales Scielo BVS- Bireme de 2001 a 2010. La prevención de riesgos ergonómicos de lesión por esfuerzo repetitivo en el equipo de enfermería tiene como objetivo mejorar las condiciones de trabajo y el conocimiento de enfermería en relación a la prevención de las enfermedades profesionales. Tres categorías surgieron del análisis textual: causas / factores de riesgo para lesiones ergonómicas, las consecuencias de los riesgos a los trabajadores y la prevención de riesgos. Así, se puso de manifiesto que entre los riesgos que reveló la organización del trabajo, los factores relacionados con el medio ambiente y la sobrecarga en los segmentos del cuerpo fueron las principales causas de absentismo, pensiones de invalidez y subsidios en el equipo de enfermería. 


\section{RESUMO}

O presente estudo objetivou conhecer os riscos ergonômicos ósteo-mioesqueléticos aos quais está exposta a equipe de enfermagem no âmbito hospitalar. Estudo descritivo-exploratório realizado através de revisão bibliográficas virtuais dos sistemas Scielo e BVS-Bireme no período de 2001 a 2010. A prevenção dos riscos ergonômicos ósteo-mioesqueléticos na equipe de enfermagem visa à melhoria das condições de trabalho e conscientização da enfermagem em relação à prevenção de doenças ocupacionais. Emergiram três categorias da análise textual: causas/fatores dos riscos ergonômicos para lesões, consequências dos riscos para os trabalhadores e prevenção dos riscos. Com isso, evidenciou-se que dentre os riscos revelados a organização do trabalho, fatores relacionados ao ambiente e sobrecarga nos segmentos corporais, foram às principais causas de afastamentos, licenças e aposentadorias por invalidez na equipe de enfermagem.

\section{ABSTRACT}

This study aimed to identify the ergonomic hazards, cumulative trauma disorders to which nursing staff in hospitals are exposed. An exploratory descriptive study was carried out by reviewing the literature and virtual systems Scielo VHL-Bireme from 2001 to 2010. The ergonomic cumulative trauma disorders prevention hazards in the nursing team aims to improve working conditions and awareness of nursing in relation to the prevention of occupational diseases. Three categories emerged from the textual analysis: causes / risk factors for ergonomic injuries, consequences of the risks to workers and risk prevention. Thus, it became clear that among the risks revealed, the organization of work, factors related to environment and overload in the body segments were the main causes of absenteeism, disability pensions and allowances in the nursing team.

\section{INTRODUCCIÓN}

En Brasil, desde los años ochenta, los accidentes de trabajo en enfermería han sido estudiados por los investigadores en un intento de determinar sus causas y proponer soluciones alternativas, que incluyen la prevención de accidentes en el lugar de trabajo. ${ }^{1,2}$

De esta manera, hay que señalar que la salud ocupacional es una disciplina en el área de la salud colectiva que tiene como objetivo centrar su atención en el proceso de la salud y la enfermedad de los trabajadores, abarcando diversas poblaciones en su relación con el trabajo. Aunque los reportes de accidentes de trabajo sean antiguos, esta es una disciplina que se encuentra todavía en construcción. ${ }^{1,2}$

Hoy en día, debido a la existencia de un mercado de trabajo altamente competitivo y a la devaluación de la mano de obra profesional en el ámbito hospitalario, es muy común encontrar profesionales de la salud, especialmente de enfermería, con más de un empleo. En consecuencia estos acaban trabajando de manera precaria y sin la seguridad necesaria, lo que afecta negativamente, tanto a la calidad del servicio como a su propia calidad de vida, acarreando para sí problemas relacionados con sus propias actividades laborales, incluidos los relacionados con su salud. ${ }^{3}$ Esta circunstancia se constituyó en la motivación principal para la realización de este estudio, con la finalidad de observar la realidad del entorno de trabajo de enfermería cuando los profesionales están expuestos a situaciones de trabajo inadecuadas relacionadas con los riesgos ergonómicos.

En este contexto, se toma como referencia la disposición reglamentaria 7 (NR-7), que tiene por objeto la elaboración y aplicación de un Programa de Salud Ocupacional, obligatorio para todas las instituciones y los empleadores que contratan trabajadores, con el objetivo de promover y preservar la salud de estos últimos. Esta ley también establece los parámetros 
mínimos y directrices a seguir en la ejecución del Programa, que pueden ser ampliados en el marco de los protocolos que regulan la negociación colectiva del trabajo. ${ }^{4}$

Los riesgos de trabajo de los profesionales de enfermería reúnen muchos factores interrelacionados, de manera que ciertos tipos de actividades y las condiciones ergonómicas inadecuadas, facilitan la aparición de tales riesgos. ${ }^{5}$

Entre los principales factores de riesgo para los trastornos mioesqueléticos se incluyen: la organización del trabajo (aumento de las horas de trabajo, exceso de horas extraordinarias, el ritmo rápido, la falta de recursos humanos), los factores ambientales (condiciones de iluminación inadecuada e insuficiente) y la posible sobrecarga que supone estrés en los segmentos corporales bajo el influjo de ciertos movimientos, por ejemplo, una fuerza excesiva en la realización de determinadas tareas, la repetición de movimientos y posturas en el desarrollo de actividades laborales. ${ }^{6}$

En este marco, se propuso como problema de investigación la pregunta siguiente: ¿cuáles son los riesgos ergonómicos a que están expuestos los profesionales de enfermería en el hospital?

Es posible establecer asociaciones entre los Trastornos MioEsqueléticos (TME) y las inadecuadas condiciones de trabajo del personal de enfermería. La exposición prolongada y continua del cuerpo a los factores de riesgo en el entorno laboral favorece el desarrollo de las enfermedades profesionales. ${ }^{7}$

\section{OBJETIVOS}

Conocer los riesgos ergonómicos mioesqueléticos a que está expuesto el personal de enfermería en los hospitales, la identificación de las causas y las consecuencias para este grupo de población.

\subsection{ANTECEDENTES}

Entre las profesiones sanitarias, la enfermería, en particular, ha sido especialmente afectada por los trastornos musculoesqueléticos. Así lo demuestran investigaciones realizadas en varios países en las que la aparición de trastornos musculoesqueléticos en trabajadores de enfermería presenta tasas de prevalencia por encima del $80 \%$. ${ }^{8,9}$

El objetivo de esta revisión es contribuir a la discusión sobre los riesgos a que están expuestos los profesionales de enfermería, lo que debe repercutir en la implementación de mejoras en las condiciones de trabajo y la calidad de vida en el sitio de trabajo.

\section{METODOLOGÍA}

Este es un estudio de tipo descriptivo-exploratorio, en el que se exponen las características de una población determinada o un fenómeno particular, con el objeto de identificar los factores que determinan o contribuyen a la ocurrencia de cierto tipo de fenómenos. ${ }^{10,11,12}$

El estudio se llevó a cabo mediante una revisión bibliográfica sistemática, basada en las obras que abordan el tema en cuestión. La recolección del material se realizó de febrero 2009 a junio 2010.

En esta revisión se presentan artículos científicos que investigan los riesgos laborales en los profesionales de enfermería. Se analizaron las publicaciones en los sistemas de colección de Scielo Biblioteca Virtual en Salud (BVS BIREME), en las fuentes (LILACS, BDENF y Medline) de 2001 a 2007. Para la búsqueda de los artículos se emplearon las palabras 
clave: el personal de salud ocupacional, enfermería, riesgos laborales, los trastornos de trauma acumulativo, la ergonomía, trastornos mioesqueléticos en enfermería.

Se destacan los términos "trabajador sano" y "enfermería" presentándose una gran cantidad de artículos sobre el tema de estudio aunque algunos en realidad estaban repetidos en bases diferentes. Estos fueron eliminados, y siempre se consideró para estas situaciones, el primer registro. Mediante el uso de las palabras "riesgos laborales" y "trastornos de trauma acumulativo" se consiguieron 3.136 documentos de manera aislada en la BVS. Se identificaron finalmente un total de 8.165 artículos para el estudio, contemplando todos los descriptores.

Para afinar la búsqueda se realizó la asociación entre descriptores, en busca de artículos que cumplieran con el propósito del estudio. Se emplearon nueve combinaciones: salud de los empleados, enfermería, riesgos laborales y trastornos de trauma acumulativo, con las que se encontraron novecientos noventa y seis artículos.

Para la selección de los artículos, se realizó una pre-lectura atenta, con la asimilación de las ideas principales para la realización después de la lectura selectiva. En esta segunda lectura se cubrió la totalidad del artículo, seleccionando el más apropiado según el propósito del trabajo.

Tabla I-Distribución de los trabajos publicados entre 2001-2007, según las fuentes virtuales en 2010.

\begin{tabular}{lccccc}
\hline \multicolumn{1}{c}{ PALAVRAS-CLAVE } & LILACS & BDENF & MEDLINE & SCIELO & TOTAL \\
\hline Enfermería + Salud del Trabajador & 1 & 1 & 0 & 1 & 3 \\
$\begin{array}{l}\text { Enfermería + Trastornos Traumáticos } \\
\text { Acumulativos }\end{array}$ & 0 & 1 & 0 & 2 & 3 \\
$\begin{array}{l}\text { Salud del Trabajador + Trastornos } \\
\text { Traumáticos Acumulativos }\end{array}$ & 1 & 0 & 0 & 0 & 1 \\
$\begin{array}{l}\text { Enfermería + Riesgos Ergonómicos } \\
\text { Enfermería + Riesgos Ergonómicos + }\end{array}$ & 0 & 1 & 0 & 2 & 3 \\
$\begin{array}{l}\text { Salud del Trabajador } \\
\text { Enfermería + Salud del Trabajador + } \\
\text { Trastornos Traumáticos Acumulativos }\end{array}$ & 0 & 0 & 0 & 0 & 0 \\
$\quad$\begin{tabular}{l} 
Total de artículos \\
\hline
\end{tabular} & $\mathbf{2}$ & $\mathbf{3}$ & $\mathbf{0}$ & $\mathbf{5}$ & $\mathbf{1 0}$ \\
\hline
\end{tabular}

Después de la selección de la literatura potencial, se llevó a cabo la lectura interpretativa que pretendía comprender los textos; esta fase sirvió para ampliar la visión sobre el tema.

A continuación, el análisis textual se orientó sobre la idea de capturar y comprender el mensaje central, sin intervenir en el contenido de los autores. En la práctica, esto implicó la realización de una serie de preguntas para el texto cuyas respuestas proporcionan el material para el análisis. Así, hubo tres categorías: las causas / factores, consecuencias y prevención de riesgos ergonómicos mio-esqueléticos.

\section{ANÁLISIS Y DISCUSIÓN DE LOS DATOS}

El debate fue organizado con el propósito de describir la información recopilada de conformidad con la organización de los temas de análisis textual: Cabe señalar que algunos artículos de corte legislativo y normativo fueron utilizados para realizar un breve resumen analítico al final de cada categoría. 


\subsection{TABLA GENERAL DE CATEGORÍAS}

En la Tabla II se ilustran los diez artículos que fueron seleccionados de acuerdo a tres categorías que surgieron del análisis textual, y se organizaron según el año de publicación.

Tabla II: Organización del trabajo, de acuerdo a las categorías de análisis textual de las obras tomadas en 2010.

\begin{tabular}{|c|c|c|c|}
\hline Autor y año & Título & Principales pruebas & Las categorías que se adapta \\
\hline $\begin{array}{l}\text { Oliveira; } \\
\text { Murofusé } \\
2001 .^{13}\end{array}$ & $\begin{array}{lr}\text { Accidentes } & \text { de } \\
\text { trabajo } & \text { y } \\
\text { enfermedad } & \\
\text { profesional: } & \\
\text { estudio sobre el } \\
\text { hospital } & \text { del } \\
\text { trabajador del } \\
\text { conocimiento de } \\
\text { los riesgos para la } \\
\text { salud en el } \\
\text { trabajo. }\end{array}$ & $\begin{array}{l}\text { Analizar los riesgos para } \\
\text { el personal de enfermería } \\
\text { en su ambiente de trabajo, } \\
\text { teniendo en cuenta el } \\
\text { desarrollo de las } \\
\text { actividades. }\end{array}$ & $\begin{array}{l}\text { Causas / factores de riesgo y } \\
\text { consecuencias de la mió } \\
\text { esqueléticos en enfermería }{ }^{(21)} \text {. }\end{array}$ \\
\hline $\begin{array}{l}\text { Reis; Rocca } \\
\text { 2003. }^{14}\end{array}$ & $\begin{array}{l}\text { Factores } \\
\text { relacionados con } \\
\text { el ausentismo por } \\
\text { enfermedad entre } \\
\text { los profesionales } \\
\text { del enfermería }\end{array}$ & $\begin{array}{lcr}\text { Absentismo } & \text { de } & \text { las } \\
\text { enfermeras } & \text { de } & \text { corta } \\
\text { duración, } & \text { con } & \text { más } \\
\text { frecuencia. } & & \\
& & \end{array}$ & $\begin{array}{l}\text { Consecuencias de los riesgos mio } \\
\text { esqueléticos en enfermería }{ }^{(22)} \text {. }\end{array}$ \\
\hline $\begin{array}{l}\text { Nishide; } \\
\text { Benatti } \\
2004 .^{15}\end{array}$ & $\begin{array}{lr}\text { Los riesgos } \\
\text { laborales de la } \\
\text { enfermería } & \text { de } \\
\text { una unidad } & \text { de } \\
\text { cuidados } & \\
\text { intensivos } & \end{array}$ & $\begin{array}{l}\text { Física y los } \begin{array}{r}\text { factores } \\
\text { ambientales }\end{array} \text { que } \\
\text { predisponen a riesgo de } \\
\text { accidentes. }\end{array}$ & $\begin{array}{l}\text { Causas I factores de riesgo y } \\
\text { prevención de la -mio esqueléticos } \\
\text { en enfermería }{ }^{(23)} \text {. }\end{array}$ \\
\hline $\begin{array}{l}\text { Robazzi; } \\
\text { Marziale } \\
2004 .{ }^{16}\end{array}$ & $\begin{array}{l}\text { El estándar de } 32 \\
\text { y sus } \\
\text { consecuencias } \\
\text { reglamentarias de } \\
\text { los trabajadores } \\
\text { enfermería }\end{array}$ & $\begin{array}{l}\text { La norma se ocupa de } \\
\text { cuestiones relativas a la } \\
\text { salud profesional y } \\
\text { conocer sus derechos y } \\
\text { cumplir sus funciones con } \\
\text { seguridad. }\end{array}$ & $\begin{array}{l}\text { Causas / Factores de Riesgo y } \\
\text { Prevención de la osteoporosis mio } \\
\text { esqueléticos en enfermería }{ }^{24)} \text {. }\end{array}$ \\
\hline
\end{tabular}




\begin{tabular}{|c|c|c|c|}
\hline $\begin{array}{l}\text { Campos } \\
\text { 2005. }\end{array}$ & $\begin{array}{ll}\text { Asistencia } & \\
\text { preventiva } & \text { de } \\
\text { enfermería a } & \text { lo } \\
\text { trabajador } & \text { de } \\
\text { enfermería } & \end{array}$ & $\begin{array}{l}\text { El aumento de las } \\
\text { condiciones de } \\
\text { prevenir lobajo y } \\
\text { laborales. }\end{array}$ & $\begin{array}{l}\text { Causas / Factores de Riesgo y } \\
\text { Prevención de la osteoporosis mio } \\
\text { esqueléticos en enfermería }{ }^{(25)} \text {. }\end{array}$ \\
\hline $\begin{array}{l}\text { Murofusé; } \\
\text { Marziale } \\
\text { 2005. }^{18}\end{array}$ & $\begin{array}{l}\text { Enfermedad del } \\
\text { sistema músculo- } \\
\text { esquelético en la } \\
\text { enfermería }\end{array}$ & $\begin{array}{l}\text { Con el fin de analizar los } \\
\text { problemas de salud } \\
\text { relacionados con el } \\
\text { sistema -mio esquelético. }\end{array}$ & $\begin{array}{l}\text { Consecuencias y prevención de } \\
\text { riesgos mio esqueléticos en } \\
\text { trabajadores de enfermería }{ }^{(26)} \text {. }\end{array}$ \\
\hline $\begin{array}{l}\text { Leite; Silva; } \\
\text { Merighi } \\
200719\end{array}$ & $\begin{array}{l}\text { Las enfermeras y } \\
\text { los trastornos } \\
\text { óseos asociados } \\
\text { al trabajo mio } \\
\text { esqueléticos }\end{array}$ & $\begin{array}{l}\text { Mayor incidencia de los } \\
\text { riesgos músculo } \\
\text { esqueléticos en una gran } \\
\text { proporción de las mujeres. }\end{array}$ & $\begin{array}{l}\text { Causas / factores de riesgo de - } \\
\text { mio esqueléticos en enfermería }{ }^{(27)} \text {. }\end{array}$ \\
\hline $\begin{array}{l}\text { Magnago, } \\
\text { Lisboa, } \\
\text { Souza; } \\
\text { Moreira } \\
200720\end{array}$ & $\begin{array}{l}\text { Los trastornos } \\
\text { músculo } \\
\text { esqueléticos en } \\
\text { trabajadores } \\
\text { enfermería: las } \\
\text { asociaciones con } \\
\text { las condiciones } \\
\text { de trabajo }\end{array}$ & $\begin{array}{l}\text { Cómo volver a pensar en } \\
\text { la organización de las } \\
\text { condiciones de trabajo. }\end{array}$ & $\begin{array}{l}\text { Causas / factores, consecuencias y } \\
\text { prevención de riesgos mió } \\
\text { esqueléticos en enfermería }{ }^{(28)} \text {. }\end{array}$ \\
\hline $\begin{array}{l}\text { Ribeiro; } \\
\text { Schimizu } \\
200721\end{array}$ & $\begin{array}{l}\text { Los accidentes de } \\
\text { trabajo entre } \\
\text { trabajadores de } \\
\text { enfermería }\end{array}$ & $\begin{array}{l}\text { El análisis de los } \\
\text { accidentes y las cargas de } \\
\text { trabajo los que están } \\
\text { expuestos. }\end{array}$ & $\begin{array}{l}\text { Causas / factores de riesgo mio } \\
\text { esqueléticos en enfermería }{ }^{(29)} \text {. }\end{array}$ \\
\hline $\begin{array}{l}\text { Monteiro; } \\
\text { AlexandreRo } \\
\text { drigues } \\
2007 .^{22}\end{array}$ & $\begin{array}{l}\text { Enfermedades del } \\
\text { aparato } \\
\text { locomotor, } \\
\text { trastornos del } \\
\text { esqueleto con el } \\
\text { trabajo, trabajo y } \\
\text { estilo de vida de } \\
\text { los trabajadores } \\
\text { en el estilo de } \\
\text { una institución de } \\
\text { salud pública }\end{array}$ & $\begin{array}{l}\text { La enfermedad causando } \\
\text { finalmente la retirada de } \\
\text { sus } \\
\text { profesionales. }\end{array}$ & $\begin{array}{l}\text { Causas / Factores de riesgo y } \\
\text { consecuencias de mio esqueléticos } \\
\text { en enfermería }{ }^{(30)} \text {. }\end{array}$ \\
\hline
\end{tabular}

\subsection{CAUSAS / FACTORES DE RIESGO MIOESQUELÉTICOS EN LOS PROFESIONALES DE ENFERMERÍA}

Entre los 10 artículos seleccionados, ocho se incluyen en esta categoría, por informar de las causas y factores de riesgo. Estos artículos demuestran claramente la fragilidad del ambiente de trabajo de Enfermería, describiendo las causas y los factores de los accidentes más comunes.

Murofusé y Oliveira (2001), realizaron un estudio en un hospital de tamaño medio con todos los funcionarios de salud, excepto los médicos. Se analizaron los riesgos inherentes a la actividad de Enfermería: esfuerzo físico, transporte y elevación manual de peso, mala postura, el trabajo nocturno, situaciones causantes de estrés psicológico, la mayoría de las veces por la disposición física, materiales inadecuados, insuficientes o defectuosos, iluminación inadecuada. ${ }^{14}$

En el segundo estudio Nishide y Benatti (2004), realizaron un estudio descriptivo, cuyo objetivo fue identificar los principales riesgos profesionales a los cuales están expuestos los profesionales de la enfermería, a partir de datos recogidos a través de entrevistas 
individuales, en donde se contemplaba el estado socioeconómico, la edad, las condiciones físicas, tales como el ambiente de trabajo y las instalaciones. Al analizar las condiciones ergonómicas de la enfermería en una sala de hospital, se encontró que la actividad de transporte de los pacientes postrados en cama fue identificada por el equipo como el más agotador desde el punto de vista físico. Se asoció este hallazgo al desgaste de muebles inadecuados y a las posturas adoptadas por el personal de enfermería. ${ }^{15}$

En el tercer estudio Robazi y Marziale (2004), se plantearon como objetivo presentar la NR 32 de salud y seguridad en el trabajo en los establecimientos de salud. Los autores reportan que la necesidad de la NR 32 proviene del alto riesgo de accidentes en el lugar de trabajo, especialmente derivados de problemas ergonómicos, por ejemplo, pisos resbaladizos. ${ }^{16}$

El cuarto artículo trabajado es el de Campos (2005), quien identificó en los discursos de los profesionales de la enfermería acciones preventivas que se recomiendan a otros profesionales de la misma área, comparándolas con las normas regulatorias. El estudio se llevó a cabo a través de búsqueda bibliográfica. Se destaca las condiciones de trabajo relacionados con la fatiga, el trabajo nocturno, las horas de trabajo, salarios bajos, problemas de dolor y sentimientos de alejamiento que conduce a trastornos del sueño, accidentes, y cambios físicos y psicofisiológicos. ${ }^{17}$

El quinto artículo es el de Leite, Silva y Merighi (2007), quienes realizaron un estudio que tuvo por objetivo describir la relación entre el profesional de enfermería y la aparición de los trastornos mioesqueléticos, relacionados con el trabajo. Se trata de un estudio teórico, donde las autoras reportaron la relación entre el proceso de trabajo y los accidentes. Hay muchos factores que favorecen la aparición de los TME: el tiempo de exposición a factores de riesgo, la organización del trabajo, la insuficiencia de recursos tecnológicos que incluye mobiliario y la existencia de equipos obsoletos, la falta de transporte de enfermos, etc. Se suma a esto la escasez de recursos humanos; el lugar donde se producen estas actividades también son importantes, así como la falta de formación relacionada con la corrección de malas posturas en la dinámica laboral. ${ }^{19}$

En el sexto estudio, Magnago, Lisboa, Souza y Moreira (2007), hicieron una revisión de artículos científicos nacionales. En este trabajo los autores combinan las condiciones TME en el trabajo, haciendo hincapié en los factores ergonómicos como una de las principales causas de estos trastornos entre el personal de enfermería. Entre las causas ergonómicas se encuentra la organización del trabajo, aumento de ritmo de carga de trabajo, escasez de mano de obra y la falta de formación, factores ambientales, mobiliario y equipos inadecuados, obsoletos, mala iluminación, deficiente temperatura, sobrecarga en determinados segmentos del cuerpo, fuerza excesiva y la repetición de movimientos. ${ }^{20}$

En el séptimo estudio, Schimizu Ribeiro (2007) constataron que en las unidades de trabajo, estos profesionales están expuestos a la diversidad y simultaneidad de cargas que pueden provocar accidentes que interactúan, teniendo en cuenta la organización y la división de trabajo de la institución. La exposición a cargas con sobrepeso en el transporte a los pacientes y la recurrencia de malas posturas, pueden causar enfermedades osteoarticulares relacionados con limitaciones físicas, ${ }^{21}$, en especial cuando se trata de exposiciones constantes y prolongadas a estas cargas

En el octavo artículo reseñado, Monteiro y Alexandre Rodrigues (2007), realizaron una investigación con 651 empleados, cubriendo 29 ocupaciones existentes en la institución, con 10 de los trabajadores de la salud. El objetivo consistía en investigar las enfermedades mio esqueléticos de los profesionales de la institución de salud y evaluar entre las personas que 
tenían diagnósticos médicos relacionados con estos problemas, la existencia de factores vinculados, teniendo en cuenta además características socio-demográficas, el trabajo y el estilo de vida. Después de un análisis descriptivo, encontraron que las mayores frecuencias se debían a insuficiencias en las herramientas de trabajo y a características funcionales desfavorables. ${ }^{22}$

En este estudio, se refleja que los riesgos ergonómicos más informados son los derivados de la organización del trabajo, ambiente de trabajo, mobiliario inadecuado, la carga de los segmentos del cuerpo debido a movimientos repetitivos y que la exposición prolongada puede aumentar el riesgo en el desarrollo de las actividades de atención directa e indirecta.

\section{RESEÑA ANALÍTICA DE LA CATEGORÍA}

Se pueden reconocer fallas en el personal de enfermería cuando se trata de evaluar los conocimientos sobre sus derechos como los definidos en la Norma 17 (NR-17), que tiene por objeto establecer los parámetros para adaptar las condiciones de trabajo a las características psicofisiológicas de los trabajadores, con el fin de proporcionar la máxima comodidad, seguridad y desempeño eficiente, y la Norma 9 (NR-9) (Ordenanza N 03 del Brasil. 214, 8 de junio de 1978), ${ }^{24}$ que establece la obligación de los empleadores y las instituciones que admiten trabajadores como empleados de planear y aplicar el Programa de Prevención de Riesgos Ambientales a fin de preservar la salud e integridad de aquellos a través de la anticipación, reconocimiento, evaluación y consiguiente control de la ocurrencia de riesgos ambientales que existan o puedan llegar a existir en el lugar de trabajo, teniendo en cuenta la protección del medio ambiente y los recursos naturales.

A través de los textos de los autores, se percibe que los profesionales de la enfermería son muy vulnerables por la persistencia de un medio ambiente de trabajo inadecuado para el desempeño de sus funciones de manera que puedan realizar una atención adecuada y segura que no ponga en riesgo su salud.

\subsection{IMPLICACIONES DE RIESGO MIOESQUELÉTICOS DE LOS TRABAJADORES DE ENFERMERÍA}

En esta categoría, se han ubicado cinco de las investigaciones en las que los autores describen las consecuencias de los riesgos ergonómicos mioesqueléticos en el equipo de enfermería en el hospital.

En el primer estudio, Murofusé y Oliveira (2001), mencionan los grupos de problemas relacionados con la organización del trabajo en el modo de producción capitalista, discutido por autores como Laurell, Dejours y Noriega, tales como el envejecimiento prematuro, el síndrome de fatiga patológica, los trastornos del sueño y la sexualidad, las situaciones de tensión crónica que causa estrés psicológico. Algunos profesionales evidencian que la rotación de turnos causa trastornos del sueño, trastornos digestivos y disturbios nerviosos, además de afectar la vida familiar y social de los trabajadores. ${ }^{13}$

En un segundo estudio Reyes y Rocca (2003), tienen como objetivo analizar los ausentismos de corto plazo en el trabajo sufrido por el personal de enfermería del hospital. Los ausentismos laborales fueron analizados teniendo en cuenta la frecuencia con que los empleados buscan la atención médica y la razón para la búsqueda. El primer análisis fue descrito como una investigación de campo, usando un abordaje de los fenómenos recurrentes, en una población de 965 empleados. En los resultados se destaca que el segundo lugar de los motivos de absentismo laboral se debe a las alteraciones mio 
esqueléticos, con $13,4 \%$ de los casos, teniendo como agravantes el agotamiento físico, psicológico y emocional, evaluando, también las condiciones de estabilidad en el empleo, analizando diferencias de acuerdo al nivel de estabilidad laboral, pues esta variable es definitiva con relación al uso de los servicios de atención de salud ${ }^{14}$

El tercer artículo realizado por Murofusé y Marcial (2005), de la Fundación Hospital de Minas Gerais, documenta un estudio de tipo descriptivo y retrospectivo, que se propone analizar los problemas de salud relacionados con el sistema mioesquelético, que se encuentran entre los equipos de profesionales de la enfermería de 23 instituciones salud atendidos por la División de Asistencia del Trabajador de la Salud, en 2002. Todos los equipos presentaron los mismos compromisos de estructura anatómica (edema superior y médula espinal) y dolor de espalda baja, siendo este el síntoma más frecuente. Con la evidencia de que la atención de los profesionales de la enfermería llevada a cabo por la División de Asistencia de los Trabajadores de la Salud fue mayor que el número de profesionales todos, en cada una de las instituciones que formaron parte del estudio, queda clara la existencia de una clase de enfermedad propia del colectivo de enfermeras y enfermeros. ${ }^{18}$

En el cuarto estudio, Magnago, Lisboa, Souza y Moreira (2007), informan el resultado de la exposición a factores de riesgo ergonómicos como problema de salud pública en Brasil, dado el gran número de retiros, pensiones de jubilación y de incapacidad de este profissional. $^{20}$

En el quinto artículo, Monteiro y Alexandre Rodrigues (2007), han reportado dolencias como resultado de la actividad profesional relacionadas con dolores frecuentes en la parte superior de la espalda o la región del cuello y de la parte baja de la espalda; dolor de espalda, este último, que se irradia por la pierna (ciática), desarrollando enfermedades músculo esqueléticas, que afectan las extremidades (brazos y piernas), y se manifiestan también como artritis reumatoide. Las enfermedades mioesqueléticas son prevalentes asociadas con la baja de los profesionales de la enfermería de sus actividades profesionales. ${ }^{22}$

\section{RESEÑA ANALÍTICA DE LA CATEGORÍA}

Se puede apreciar en el estudio que el personal de enfermería tienen una mayor incidencia de absentismo de sus actividades que otros profesionales de la salud, debido a los accidentes de trabajo o a la presencia de algún tipo de enfermedad ocupacional; situación que trae consigo el incremento de miedos y temores derivados de la inestabilidad profesional por posible destitución o separación del empleo, debida precisamente a la incapacidad laboral combinada con recurrentes ausentismos en las instituciones.

El Decreto N 1. GM 12506 de julio 2005 define la política para el personal de la salud en el marco del Sistema Unificado de Salud (SUS), que establece que cualquier política de salud de los trabajadores tiene el propósito de promover la salud y la reducción de morbilidad y mortalidad a través de actividades integradas, intra e intersectoriales, de forma continua, con acción sobre los determinantes de los problemas de salud derivados de los modelos y procesos de desarrollo, con la participación de todos los sujetos sociales involucrados. Las acciones de salud desarrolladas por el SUS se organizan en todos los niveles de atención, con las siguientes directrices: atención integral de salud, involucrando la promoción de ambientes saludables, prevención de lesiones relacionadas con el trabajo, la atención de salud integral y la adecuación y ampliación de la capacidad institucional. ${ }^{25}$ 


\subsection{PREVENCIÓN DE RIESGOS MIOESQUELÉTICOS EN LOS TRABAJADORES DE ENFERMERÍA}

Se describen en esta categoría, los cinco estudios que abordan la prevención de los riesgos mioesqueléticos entre el personal de enfermería.

En el primer artículo Nishide y Benatti (2004), señalan como prevención el simple hecho de la organización del espacio físico, el almacenamiento de equipo y mobiliario, así como la reparación de equipos y mobiliario con problemas técnicos, evitando así la exposición de los trabajadores a estos riesgos. ${ }^{15}$

En el segundo documento, Robazzi y Marziale (2004), señalan la importancia de la Regla 32 (NR-32) de la ley federal de Brasil, ya que trata temas relacionados con la salud del personal de enfermería en su ambiente de trabajo; la norma permite a estos profesionales conocer sus derechos y ejercer sus funciones con seguridad. ${ }^{16}$

En el tercer estudio, Campos (2005), pone de relieve el hecho de que para revertir el alejamiento del personal de enfermería es necesario cambiar la forma de actuar, ya que estos profesionales están orientados a la preservación de la vida, la integridad física y moral de los pacientes, sin poder resolver sus propios problemas de salud como este relacionado con las enfermedades ocupacionales. ${ }^{17}$

En el cuarto estudio Murofusé y Marziale (2005), informan sobre la necesidad de una mayor atención que debe darse a las posturas corporales en la ejecución de las actividades, la garantía de condiciones adecuadas en el mobiliario pues sólo entonces es posible pensar que la atención médica pudiera descender bruscamente. ${ }^{18}$

En el quinto estudio Magnago, Lisboa, Souza y Moreira (2007), señalan la importancia de la planificación, la adquisición de materiales y programas de formación para los profesionales de enfermería como un medio de prevenir numerosas ausencias laborales debido a enfermedades ocupacionais. ${ }^{20}$

En la sexta ponencia Schimizu Ribeiro (2007), llama la atención sobre la importancia de que las instituciones realicen inversiones en la formación de los profesionales para que estos adopten posturas correctas y para que la modernización de los equipos sea una política permanente, reduciendo así los alejamientos laborales causados por tales trastornos profesionales. . $^{21}$

\section{RESEÑA ANALÍTICA DE LA CATEGORÍA}

Se identificó en esta categoría, que las instituciones deben realizar inversiones en capacitación para que los empleados adopten posturas correctas y los equipos sean permanentemente modernizados y de esta manera, evitar el desgaste causado por las cargas fisiológicas. Para lograr condiciones adecuadas y seguras el servicio de educación continua necesita operar con el personal de enfermería a fin de reconocer la importancia de la prevención de accidentes y promoción de la salud en el trabajo.

En realidad, los trabajadores deben ser rigurosamente animados a mantener su salud en el trabajo, pero también hay necesidad de adaptar el trabajo al hombre, en particular en la concepción y diseño de los puestos de trabajo, de los equipamientos y en los diseños de los procesos y métodos de trabajo. Esta necesidad aún existe en la legislación vigente.

Con la consolidación de las leyes laborales con respecto a la seguridad y salud en el trabajo, se dictaron diversas normas reglamentarias encaminadas a la protección de los trabajadores en su ambiente de trabajo, el énfasis en la ley 6. Art. 514/77. 200: faculta al 
Ministerio de Trabajo para crear disposiciones adicionales a las normas, teniendo en cuenta las peculiaridades de todas las actividades de trabajo en los distintos sectores, en particular con respecto a la protección de los empleados a la exposición productos químicos peligrosos, radiaciones ionizantes y no ionizantes, el ruido, la vibración y agitación o el medio ambiente anormal, la presión en el trabajo, especificando las medidas adecuadas para eliminar o reducir estos efectos; el límite superior en el momento de la exposición, la intensidad de la acción o sus efectos en el cuerpo, de control médico, los límites de edad, control permanente de los lugares de trabajo y otros requisitos que se consideren necesarios. ${ }^{26}$

\section{CONCLUSIÓN}

En primer lugar, este estudio encuentra que el equipo de enfermería enfrenta condiciones laborales inadecuadas en su medio ambiente de trabajo, como un desafío en su rutina diaria de asistencia y cuidado, que puede dar lugar a riesgos ergonómicos. Los principales fueron la organización del trabajo (jornada excesiva, déficit profesional), los factores relacionados con el medio ambiente (mobiliario y equipos inadecuados y obsoletos), y sobrecargas en los segmentos corporales.

Estos riesgos generan gran problema de salud pública debido al absentismo, los permisos y el retiro por discapacidad. Cabe señalar que el grupo de enfermería puede necesitar una legislación específica para la protección y seguridad en el trabajo, y por lo tanto, resulta muy importante que el empleado asuma también sus funciones como ciudadano en el cumplimiento de sus deberes, pero también en la reafirmación de sus derechos, especialmente los relacionados con su seguridad.

Entre los desafíos que enfrentan en este estudio destaca el bajo número de publicaciones en área específica de la enfermería sobre el tema. Se considera muy importante que los profesionales y estudiantes de enfermería puedan profundizar en el estudio de esta problemática, con el objeto de una difusión más amplia y una mayor comprensión.

Se sugiere la realización de futuros estudios, cuyas líneas lleven a la normalización de los métodos de investigación sobre la evaluación de la exposición y de afectaciones mio esqueléticas con el objetivo de ofrecer los medios más seguros para proponer medidas de prevención y promoción de la salud de la enfermería.

\section{REFERÊNCIAS}

1 Sêcco IAO, Robazzi MLCC, Gutierrez PR, Matsuo T. Acidentes de Trabalho e Riscos Ocupacionais no dia-a-dia do trabalhador hospitalar: desafio para a Saúde do Trabalhador. Rev. Bras. Enf. 2002; (4). Disponível em: http://www.ccs.uel.br/espacoparasaude/v4n1/doc/hospital.htm .

${ }^{2}$ Ergonomia, Conceitos, Origens e Cronologia, 1999-2001. [Internet]. Disponível em: http://www.ergonomia.com.br/htm/historico.htm

${ }^{3}$ Castro MR, Farias SNP. A produção cientifica sobre riscos ocupacionais a que estão expostos os trabalhadores de enfermagem. Rev. Esc. Enf. Anna Nery. 2008; 12(2): 364-69.

${ }^{4}$ Brasil. Ministério do trabalho e emprego. Norma regulamentadora 7 (NR-7). Dispõe sobre o programa de controle médico de saúde ocupacional - EPI de 08 de junho de 1978. Diário oficial da união de 06/07/1978. Disponível em: http://www.mte.gov.br/legislacao/normas regulamentadoras/nr 07 at.pdf. Acessado em: 10/01/2010. 
${ }^{5}$ Comélio ME, Alexandre NMC. Avaliação de uma cadeira de banho utilizado em ambiente hospitalar: uma abordagem ergonômica. Rev. Bras. Enferm. 2005; 58(4) 405-410.

${ }^{6}$ Mauro MYC, Cupello AJ, Mauro CCC. O trabalho de enfermagem hospitalar: uma visão ergonômica. . [Internet]. [citado em 2009 fev 15]. Disponível em: URL: http://www.alass.org/es/actas/80-BR.htlm

${ }^{7}$ Gurgueira GP, Alexandre NMC, Filho HRC. Prevalência de sintomas musculoesqueléticos em trabalhadores de enfermagem. Rev. Latino Americana de Enfermagem. 2003; 11(5): 608-13.

8 Botha WE, Bridger RS. Anthropometric variability, equipment usuability and musculoskeletal pain in a group of nurses in the Western Cape. Appl Ergon 1998; 29: 481 90.

${ }^{9}$ Ando S, Ono Y, Shimaoka M, Hiruta S, Hattori Y, Hori F, Takeuchi Y. Associations of self estimated workloads with musculoskeletal symptoms among hospital nurses. Occup Environ Med. 2000; 57: 211-6.

${ }^{10}$ FIGUEIREDO NMA. Método e metodologia na pesquisa científica. São Paulo: Yendis, $3^{\text {a }}$ ed; 2008.

${ }^{11}$ Chaves MA. Projeto de pesquisa guia prático para monografia. $4^{a}$ ed. Rio de Janeiro: Wak ;2007.

${ }^{12}$ Severino AJ. Metodologia do trabalho cientifico. 2ª̃ed. São Paulo: Cortez; 2002.

${ }^{13}$ Oliveira BRG, Murofusé NT. Acidentes de trabalho e doença ocupacional: estudo sobre o conhecimento do trabalhador hospitalar dos riscos à saúde de seu trabalho. Rev.latino Americana enfermagem. 2001; 9(1):109-115.

${ }^{14}$ Reis RJ, Rocca PF, Silveira AM, Bonilla IML, Ginéc NA, Martín M. Fatores relacionados ao absenteísmo por doença em profissionais de enfermagem. Rev Saúde Pública. 2003; 37 (5): 616-23.

${ }^{15}$ 23-Nishide VM, Benatti MCC. Riscos ocupacionais entre trabalhadores de enfermagem de uma unidade de terapia intensiva. Rev. Esc. Enferm. USP. 2004, 38 (4): 406:14.

${ }^{16}$ Robazzi MLCC, Marziale MHP. A norma regulamentadora 32 e suas implicações sobre trabalhadores de enfermagem. Rev. Latino-Am. Enfermagem. 2004; 12 (5).834-6.

17 Campos ALA, Gutierrez PSG. Assistência preventiva do enfermeiro ao trabalhador de enfermagem. Rev. Bras. Enferm. 2005; 58 (4):458-461.

18 Murofusé NT, Maziale MHP. Doenças do sistema ostemuscular em trabalhadores enfermagem. Rev. Latino Americana. 2005; 13 (3): 364-73.

${ }^{19}$ Leite PC, Silva A, Merighi MAB. A mulher trabalhadora de enfermagem e os distúrbios osteomusculares relacionados ao trabalho. Rev. Esc. Enferm. USP. 2007; 2007; 41(2):28791.

${ }^{20}$ Magnago TSBS, Lisboa MTL, Souza IEO, Moreira MC. Distúrbio músculo esquelético em trabalhadores de enfermagem: associações com condições de trabalho. Rev. Bras. Enferm. 2007; 60 (6):701-705.

${ }^{21}$ Ribeiro EJG, Shimizu HE. Acidentes de trabalho com trabalhadores de enfermagem. Rev. Bras. Enferm. 2007; 60 (5): 535-540.

${ }^{22}$ Monteiro MS, Alexandre NMC, Rodrigues CM. Doenças músculo-esqueléticas, trabalhoesqueléticas, trabalho e estilo de vida entre trabalhadores de estilo de uma instituição pública de saúde. Rev. Esc. Enferm. USP. 2006, 40(1):20-5.

${ }_{23}$ Brasil. Ministério do trabalho e emprego. Norma regulamentadora no 17 (NR-17). Estabelece parâmetros que permitam a adaptação das condições de trabalho às características psicofisiológicas dos trabalhadores de 08 de junho de 1978. Diário oficial união de 06/07/1978. Disponível em:

http://www.mte.gov.br/legislacao/normas regulamentadoras/nr 17.pdf. Acessado em $10 / 01 / 2010$

${ }^{24}$ Brasil. Ministério do Trabalho e Emprego. Norma regulamentadora nำ (nr-9). Dispõe sobre o Programa de prevenção de riscos ambientais de 08 de junho de 1978. Diário Oficial 
da União de 06/07/1978. Disponível em:

http://www.mte.gov.br/legislacao/normas_regulamentadoras/nr_09_at.pdf . acessado em 10/01/2010.

${ }^{25}$ Brasil. Ministério da Saúde. Portaria no 1.125/GM de 6 de julho de 2005. Disponível em: http://dtr2001.saude.gov.br/sas/PORTARIAS/Port2005/GM/GM-1125.htm 24 de maio de 2009. Acesso em 15/11/2010.

26 Brasil. Presidência da República. Lei no 6.514. Altera o Capítulo V do Titulo II da Consolidação das Leis do Trabalho, relativo à segurança e medicina do trabalho e dá outras providências de 22 de dezembro de 1977. Diário oficial da união de 23/12/1977. Disponível em: http://www.planalto.gov.br/ccivil_03/Leis/L6514.htm.Acessado em: 10/01/2010. 